\title{
Labial soft tissue volume evaluation of different techniques for ridge preservation after tooth extraction: a randomized controlled clinical trial
}

Schneider, David ; Schmidlin, Patrick R ; Philipp, Alexander ; Annen, Beat M ; Ronay, Valerie ; Hämmerle, Christoph H F ; Attin, Thomas ; Jung, Ronald E

\begin{abstract}
OBJECTIVE: To volumetrically evaluate soft tissue changes of different ridge preservation techniques compared to spontaneous healing 6 months after tooth extraction. MATERIALS AND METHODS: In each of 40 patients, one single-rooted tooth was extracted and four treatment modalities were randomly assigned to the following groups $(\mathrm{n}=10 \mathrm{each})$ : A) B-tricalcium-phosphate-particles with a polylactid coating (B-TCP), B) demineralized bovine bone mineral with $10 \%$ collagen covered with a collagen matrix (DBBM-C/CM), C) DBBM with $10 \%$ collagen covered with an autogenous soft tissue punch graft (DBBM-C/PG), D) spontaneous healing (control). Impressions were obtained before extraction and 6 months later, casts were digitized and volumetric changes at the buccal soft tissues were determined. One-way anova was performed and pair-wise Wilcoxon rank sum test with Bonferroni-Holm method was applied for comparison of differences between two groups. RESULTS: After 6 months, horizontal contour changes accounted for $-1.7 \pm 0.7 \mathrm{~mm}(\mathrm{~A}),-1.2 \pm 0.5 \mathrm{~mm}$ (B), $-1.2 \pm$ $0.7 \mathrm{~mm}(\mathrm{C})$ and $-1.8 \pm 0.8 \mathrm{~mm}(\mathrm{D})$. None of the group comparisons reached statistical significance. CONCLUSIONS: Six months after tooth extraction all groups revealed a horizontal volume change in the buccal soft tissue contour. Application of DBBM-C/CM or DBBM-C/PG reduced the amount of volume resorption compared to B-TCP or spontaneous healing without reaching statistically significant difference.
\end{abstract}

DOI: https://doi.org/10.1111/jcpe.12246

Posted at the Zurich Open Repository and Archive, University of Zurich

ZORA URL: https://doi.org/10.5167/uzh-96099

Journal Article

Accepted Version

Originally published at:

Schneider, David; Schmidlin, Patrick R; Philipp, Alexander; Annen, Beat M; Ronay, Valerie; Hämmerle, Christoph H F; Attin, Thomas; Jung, Ronald E (2014). Labial soft tissue volume evaluation of different techniques for ridge preservation after tooth extraction: a randomized controlled clinical trial. Journal of Clinical Periodontology, 41(6):612-617.

DOI: https://doi.org/10.1111/jcpe. 12246 


\section{Labial soft tissue volume evaluation of different techniques for ridge preservation after tooth extraction: A randomized controlled clinical trial}

David Schneider ${ }^{b^{*}}$, Patrick R. Schmidlin ${ }^{a^{*}}$, Alexander Philipp ${ }^{a, c}$, Beat M. Annen ${ }^{c}$, Valerie Ronay ${ }^{a}$, Ronald E. Jung ${ }^{b}$

a Clinic of Preventive Dentistry, Periodontology and Cariology, Center of Dental Medicine, University of Zurich, Switzerland

${ }^{\mathrm{b}}$ Clinic of Fixed and Removable Prosthodontics and Dental Material Science, Center of Dental Medicine, University of Zurich, Switzerland

${ }^{\mathrm{C}}$ Private Practice, Zurich, Switzerland

* Both authors with an asterisk contributed equally to this study and share first authorship

Key words: Ridge preservation, bone regeneration, CAD/CAM, human, graft material, bone substitute, RCT

Running title: Volume evaluation of ridge preservation

Address for correspondence:

Prof. Dr. Patrick R. Schmidlin

Clinic of Preventive Dentistry, Periodontology and Cariology

Center of Dental Medicine, University of Zurich

Plattenstrasse 11

$\mathrm{CH}-8032$ Zurich, Switzerland

Phone: +4144634 3251

Fax: +41446344305

e-mail: patrick.schmidlin@zzm.uzh.ch 


\section{Abstract}

Objective: To volumetrically evaluate soft tissue changes of different ridge preservation techniques compared to spontaneous healing 6 months after tooth extraction.

Materials and Methods: In each of forty patients, one single-rooted tooth was extracted and four treatment modalities were randomly assigned to the following groups ( $n=10$ each): A) ß-tricalcium-phosphate-particles with a polylactid coating (ßTCP), B) demineralized bovine bone mineral with $10 \%$ collagen covered with a collagen matrix (DBBM-C/CM), C) DBBM with $10 \%$ collagen covered with an autogenous soft tissue punch graft (DBBM-C/PG), D) spontaneous healing (control). Impressions were obtained before extraction and 6 months later, casts were digitized and volumetric changes at the buccal soft tissues were determined. One-way ANOVA was performed and pair-wise Wilcoxon rank sum-Tests with Bonferroni-Holm method was applied for comparison of differences between two groups.

Results: After 6 months, horizontal contour changes accounted for $-1.7 \pm 0.7 \mathrm{~mm}$ (A), $-1.2 \pm 0.5 \mathrm{~mm}(B),-1.2 \pm 0.7 \mathrm{~mm}(\mathrm{C})$ and $-1.8 \pm 0.8 \mathrm{~mm}(\mathrm{D})$. None of the group comparisons reached statistical significance.

Conclusions: Six months after tooth extraction all groups revealed a horizontal volume change of the buccal soft tissue contour. Application of DBBM-C/CM or DBBM-C/PG reduced the amount of volume resorption compared to B-TCP or spontaneous healing without reaching statistical significant difference. 


\section{Clinical relevance}

Scientific rationale for the study: Tooth extraction results in several clinical, histological and morphological changes, which may hamper implant therapy especially in terms of a negative influence of the esthetic outcomes after soft tissue remodeling. Ridge preservation procedures could possibly counteract this loss of volume.

Principal findings: The dimensional changes following tooth extraction and subsequent ridge preservation varied. The application of a slowly resorbing biomaterial covered with either autogenous soft tissue punch or a collagen matrix revealed some advantages, however, they were not statistically significant.

Practical implications: Ridge preservation techniques applying slowly resorbing biomaterials with a socket seal and without raising a flap can only partially maintain the original anatomical soft tissue contours. For a $100 \%$ preservation of the soft tissue contour a flap elevation and an augmentation procedure to the buccal aspect of the alveolar ridge needs to be considered. 


\section{Introduction}

Immediately following tooth extraction biological processes are initiated, which lead to bone resorption and subsequent soft tissue contour changes (Amler, et al. 1960) (Pietrokovski \& Massler 1967). A recent systematic review on post-extractional alveolar hard and soft tissue alterations in humans reported about $29-63 \%$ horizontal changes after 6-7 months (Tan, et al. 2012). The percentage of vertical changes were less pronouced ranging from 11-22\% (Tan, et al. 2012).

In order to overcome these alterations a variety of clinical and preclinical studies using different kind of bone substitute materials with or without flap evaluation have been performed (Botticelli, et al. 2004) (Araujo, et al. 2008) (Fickl, et al. 2008) (Jung, et al. 2013). Another current systematic review outlined the potential benefit of alveolar ridge preservation therapies resulting in significantly less vertical and horizontal contraction of the alveolar bone crest compared to spontaneous healing (Vignoletti, et al. 2012). Regarding soft tissue remodeling, a previously published clinical study showed that different alveolar ridge preservation techniques were not able to entirely compensate for alveolar ridge reduction as well when evaluating optically scanned cast models (Thalmair, et al. 2013). In general, there is very limited data in dental literature available on soft tissue volume changes after tooth extraction using different techniques for alveolar ridge preservation.

Hence, the present study was initiated to evaluate the volumetric changes of the soft tissue contour using different ridge preservation techniques compared to spontaneous healing within the first 6 months after tooth extraction. It is based on a clinical data set of a recently published randomized clinical trial, which focused on the assessment of radiographic changes of the alveolar ridge following application of different ridge preservation techniques (Jung, et al. 2013). 
The hypothesis was that spontaneous healing would result in more pronounced contour changes as compared to ridge preservation techniques. 


\section{Materials and Methods}

\section{Study design}

The present study reports on the clinical volumetric data of a randomized, controlled, clinical investigation, where the radiographic results from cone beam computer tomography scans (CBCT) have already been published (Jung, et al. 2013). All clinical procedures were performed at the Clinic of Preventive Dentistry, Periodontology and Cariology, and at the Clinic for Fixed and Removable Prosthodontics and Dental Material Science Center of Dental Medicine, University of Zurich, Switzerland. The clinical study protocol and all procedures and materials were approved by the local ethical committee of the Canton of Zurich (Ethikkommission des Kanton Zürich, Ref.Nr. StV 06/12). Informed consent was obtained from all patients prior to the start of the study.

\section{Participants}

The primary inclusion criterion was the necessity of at least one upper or lower asymptomatic front tooth or premolar that needs to be extracted. The diagnosed indications for tooth extractions were mainly caries, endodontic complications (e.g. root fracture) and periodontitis. Teeth were also extracted for orthodontic and prosthetic reasons. Only healthy patients with an adequate oral hygiene (bleeding on probing $\leq 20 \%$; Plaque index $\leq 20 \%$ ) were included in this study. As a prerequisite for inclusion in the study, patients had to be systemically healthy and not smoke more than 20 cigarettes/day.

Forty patients fulfilled all the inclusion criteria and were subsequently enrolled in the study. 


\section{Treatment protocol and interventions}

Prior to tooth extraction and before anesthetizing the area, a silicone impression (President, Coltene, Altstätten Switzerland) was taken of the relevant jaw segment. Subsequently, cast models were fabricated from dental stone (camtec-rock, Picodent, Wipperfürth, Germany)

The subsequent treatment protocol has previously been described in detail (Jung et al 2013). In brief, after gentle tooth extraction and measuring the height of the buccal bone plate participants were excluded if more than $50 \%$ of the buccal bone height was not present. The height of the buccal bone after extraction of the tooth was assessed by linear measurements from the gingival margin to the buccal alveolar crest and to the most apical part of the extraction socket using a periodontal probe. Thereafter, patients were randomly assigned to one of three test ridge preservation techniques or the control group according to a computer-generated randomization list ( $\mathrm{n}=10$ per group):

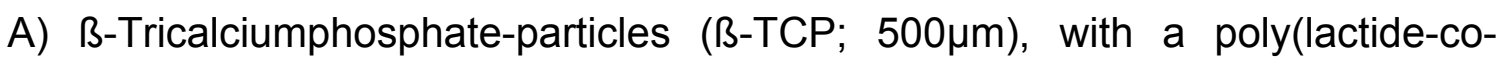
glycolide) coating (easy-graft ${ }^{\circledR}$, Degradable Solutions AG, Schlieren, Switzerland) 1-2 mm below the bone crest without any further treatment at the soft tissue level according to the instructions of the manufacturer.

B) Demineralized bovine bone mineral with $10 \%$ collagen (Bio-Oss ${ }^{\circledR}$ Collagen, Geistlich Pharma AG, Wolhusen, Switzerland) at bone level and application of a collagen matrix (CM; Mucograft ${ }^{\circledR}$ Geistlich Pharma AG, Wolhusen, Switzerland) at the soft tissue level (DBBM-C/CM).

C) DBBM-C (Bio-Oss ${ }^{\circledR}$ Collagen, Geistlich Pharma AG, Wolhusen, Switzerland) at the bone level and application of an autogenous soft tissue punch graft (PG) at the soft tissue level (DBBM-C/PG). 
D) For the control group no further treatment was applied and the coagulum within the socket was left open for spontaneous healing.

The application of the regenerative materials has been descripted in the previous study on the radiographic data (Jung, et al. 2013).

Postextraction the patients were instructed to rinse twice daily with $0.2 \%$ Chlorhexidine (Kantonsapotheke Zürich), and received pain killer medication (Mefenacid, Streuli Pharma, Uznach, Switzerland) and antibiotics (Amoxicillin Sandoz, Sandoz, Basel, Switzerland) for 5 days. All patients were recalled at 7-10 days for a check-up and suture removal (groups DBBM-C/PG and DBBM-C/CM). Patients followed then their individual maintenance program according to the individual periodontal and caries risk assessment.

\section{Follow-up}

Six months after extraction, another impression was taken using silicone material and cast models were fabricated from dental stone according to the procedure at baseline.

In addition to the silicone impressions, cone beam computer tomography (CBCT) scans were obtained immediately after treatment and 6 months thereafter using a KaVo 3D exam CBCT scanner (KaVo, Biberach an der Riss, Germany) as described in the previously published study (Jung, et al. 2013).

Soft tissue volume analyses 
For the evaluation the soft tissue contour changes, all plaster models were digitized using an optical scanner (Imetric 3D GmbH, Courgenay, Switzerland). The digital data of these models were imported into a software for volumetric analysis (Swissmeda/SMOP, Zürich, Switzerland). Digital cast models representing the different time-points during the treatment were superimposed using the best-fit algorithm at unchanged tooth surfaces. The relevant area for the measurements of volume changes was defined according to previous studies on ridge contour alterations (Schneider, et al. 2011) (Fickl, et al. 2009) (Thoma, et al. 2010). The area was defined by the mesial and distal papillary midline and the mucogingival line (Figure 1). Where necessary, the area of interest was shifted 1-2 mm in apical direction, to compensate for the underlying surface loss due to vertical resorption in the most coronal tissue after remodeling during the healing phase. Not shifting the area in these cases would have resulted in non-readable measurements due to an inevitable lack of a superimposable surface.

Since this area was different in size for each site, the mean change of volume per area was calculated, resulting in a distance in labial direction. Thereby, different sites could be directly compared in terms of volume changes irrespective of their size and the size of the measured area.

Statistical analysis:

Descriptive statistics were applied to describe the means, medians, standard deviations, quartiles, minimum and maximum values for each treatment group. Kruskal-Wallis non-parametric one-way analysis of variance was performed to identify differences between groups. In addition, the pair-wise Wilcoxon rank sum- 
Tests with Bonferroni-Holm method was applied for comparison of differences between two groups.

For all analyses, the level of significance was set at $p<0.05$. Number needed to treat to identify a difference of $1 \mathrm{~mm}$ in distance between each treatment group was calculated based on differences of the means.

Sample size calculation was described and discussed in the previous study (Jung, et al. 2013) where the power analysis revealed a minimum sample size of approximately 240 patients to be included aiming to achieve $81 \%$ power to detect a $0.5 \mathrm{~mm}$ difference with standard deviations of $1 \mathrm{~mm}$. However, this sample size was found to be far beyond the scope and the feasibility of this study. Therefore, a realistic sample size of 36 subjects was selected for power analysis calculation: Based on group sizes of nine patients, a significance level of $5 \%$ and a population within-group standard deviation of $0.5 \mathrm{~mm}$ a parametric one-way ANOVA will be able to detect with $52 \%$ power a true mean difference of $0.5 \mathrm{~mm}$ between the control group and the three test groups. Put differently, the study could detect a true mean difference of $0.67 \mathrm{~mm}$ with $80 \%$ power given population within-group standard deviations of $1 \mathrm{~mm}$. With the results of the present study a power of 0.46 could be achieved. 


\section{Results}

\section{Patients}

The treatments were performed according to the clinical protocol revealing no postoperative complications at any included site. All patients completed the study and passed the 6-month follow-up.

From a total of 40 maxillary and mandibular extraction sites, 23 involved bicuspids, 8 laterals incisors, 6 central incisors and 3 canines. Sites were equally distributed among the four treatment modalities and were not suspected to influence the treatment outcomes.

36 patients were included for volumetric data analysis. Data of 4 patients ( 1 from test 1 group, one from test 2 group and 2 from control group) could not be obtained due to insufficient quality of impressions and/or casts or changes of reference points needed for superimposition during the treatment period. These patients had to be excluded from analysis.

Analysis of soft tissue contour changes

The mean horizontal changes in labial/buccal direction of the soft tissue contour are depicted in figure 2 and table 1.

The most pronounced resorption was found in the control group $D(-1.8 \mathrm{~mm} \pm 0.8$ $\mathrm{mm})$, followed by test group $A(-1.7 \pm 0.7 \mathrm{~mm})$. In groups $B(-1.2 \pm 0.7 \mathrm{~mm})$ and $C(-$ $1.2 \pm 0.7 \mathrm{~mm}$ ) the mean distances were less, however, the differences between groups were not statistically significant (table 2 ).

The number needed to treat (NNT) to achieve a difference in mean distance of $1 \mathrm{~mm}$ is displayed in table 3. NNT refers to the calculated average number of patients who need to be treated to benefit from one treatment modality over the other, i.e. who 
have 1 millimeter less volume change. The highest NNT was observed between groups B and C (NNT=100). An NNT of 1.9 and 1.6 were found for the comparison between $A$ and $B / C$ and $D$ and $B / C$, respectively. This means that almost every second patients treated with modalities $B$ or $C$ would exhibit less than $1 \mathrm{~mm}$ of contour shrinkage as compared to modalities A and D.

The mean horizontal changes based on cone-beam CT data in labial/buccal direction of the different treatment modalities are displayed in figure 3 (Jung et al. 2013). The mean changes at the bone level in groups $A, B, C$ and $D$ accounted for $3.3 \pm 1.4 \mathrm{~mm}, 0.7 \pm 0.4 \mathrm{~mm}, 0.9 \pm 0.5 \mathrm{~mm}$ and $2.2 \pm 0.8 \mathrm{~mm}$, respectively. 


\section{Discussion}

This study was performed to compare the effect of different techniques for ridge preservation with spontaneous healing on a soft tissue contour level. After 6 months, spontaneous healing showed the most pronounced reduction of the labial ridge contour (mean $-1.8 \mathrm{~mm}$ ) closely followed by the ß-TCP/PLGA group (mean -1.7 $\mathrm{mm})$. Smaller reductions were found for the DBBM-C/Collagen matrix $(-1.2 \mathrm{~mm})$ and the DBBM-C/Punch (-1.2 mm) groups. The differences between all latter treatments were not statistically significant.

This finding is in accordance to a recent study which showed that alveolar ridge preservation using xenogenic bone substitute (pre-hydrated collagenated corticocancellous porcine bone) with or without a free gingival graft was not able to entirely compensate for the alveolar ridge reduction. Covering the orifice of the extraction socket using a free gingival graft, with or without application of a filler material, was able to somewhat limit the post-operative external contour shrinkage (Thalmair, et al. 2013). The present study showed a similar trend.

A study in dogs assessing volumetrically the occurring alterations of the ridge contour after alveolar ridge preservation with and without and buccal overbuilding also showed - in accordance to our findings - no statistically significant differences with regard to the buccal volume per area among the treatment groups four months after tooth extraction (Fickl, et al. 2009). The observed resorption rate was in the range between 1.5 and $2.0 \mathrm{~mm}$, which was also within the range of the present findings.Both studies used the same methodology in assessing the volume differences.

A recent systematic review summarized the quantitative data of alveolar ridge preservation in terms of bone changes (Horvath, et al. 2013). Although a meta- 
analysis was not possible due to the clinical heterogeneity of the studies, a significantly smaller average loss of clinical alveolar ridge width ranging between 1.0 and $-3.5 \pm 2.7 \mathrm{~mm}$ was found in treated sites/groups as compared to the untreated control sites/groups with a range between -2.5 and $-4.6 \pm 0.3 \mathrm{~mm}$. The mean change in clinical alveolar ridge height varied between $+1.3 \pm 2.0$ and $-0.7 \pm$ $1.4 \mathrm{~mm}$ in treated and between $-0.8 \pm 1.6$ and $-3.6 \pm 1.5 \mathrm{~mm}$ in the control sites/groups. Again results were statistically significant favouring sites with a ridge preservation approach. Compared to the soft tissue alterations in the present study, more distinct differences could be found on a bone level (Jung, et al. 2013).

Therefore, soft tissue changes do not seem to completely follow the changes at the alveolar bone level as recorded in the same patients in the previous study (Jung, et al. 2013). Although not based on a hard statistical facts due to different methodology, the comparison of the corresponding box-plots suggests that larger resorption rates of the bone may lead to less accentuated remodeling of the soft tissue contour. A reason might be that in the first study the region of interest was positioned in areas that were considered optimal for the analysis of alveolar ridge dimensions which was one, three and five millimeters from the initial alveolar crest. The most interesting and relevant areas for soft tissue contour alterations, however, are situated more coronally in the area of the former gingival margin before tooth extraction. Areas within the non-keratinized tissue had to be excluded from the analysis due to the mobility and instability of the surface, which did not allow for reproducible measurements. Therefore, the localization of the optimal area for bone measurements might potentially be more apically located as compared to the actually adequate area for soft tissue contour measurements. This topographic discrepancy can lead to different measurements between bone and soft tissues. 
Nevertheless, focusing on the soft tissue and bone-remodeling pattern, both parameters followed the same resorption trend.

A further methodological limitation of the present study was the fact that the measured area of interest had to be shifted apically if on the follow-up model the underlying surface was lost due to extensive vertical resorption. Hence, the amount of soft tissue volume loss taking place within the most critical area could methodologically not be captured and is therefore masking part of the volume alterations. This limitation masking part of the volume changes is mainly affecting sites revealing more pronounced vertical loss. According to the previous study assessing the bone changes this is true for the $ß$-TCP/PLGA sites (group $A$ ) and the control sites (group D) (Jung, et al. 2013). This could be an additional explanation for the smaller and not significant differences when comparing groups $\mathrm{B}$ and $\mathrm{C}$ with groups $A$ and $D$.

Power analysis revealed that there was a lack of power in the present study due to the small sample size, which may be another reason that not more differences between groups were found. In addition to the relatively small sample, the influence of the soft tissue biotype and the position of the site in the alveolar arch (e.g. bicuspids vs. incisors) have not been investigated. These limitations should be considered for future research, which should include a higher sample size with more homogenous sites and a smaller number of treatment groups.

Based on the present and previous data, clinical applications of ridge preservation techniques should be further evaluated regarding their cost-benefit ratio. On a soft tissue level, the treatment modality may be considered less critical in terms of contour alterations than focusing on the preservation of alveolar bone. Depending on the subsequent treatment plan after extraction (e.g. implant therapy versus 
bridge/pontics) the use of adequate materials and methods should be carefully considered. Furthermore, long-term performance of such treatments is of outmost importance to ensure an optimal stability of the respective tissues. Studies assessing these changes after a time period of 6 months and longer are still scarce. 


\section{Conclusions}

Six months after tooth extraction all groups revealed a horizontal volume change of the buccal soft tissue contour. Application of DBBM-C/CM or DBBM-C/PG reduced the amount of volume resorption compared to B-TCP or spontaneous healing without reaching statistical significant difference.

The possible influence of materials and techniques on the long-term outcomes of subsequent implant therapy or conventional prosthodontics has to be further investigated and plays an important role when selecting the appropriate measures.

\section{Conflict of interest and source of funding statement}

The present study was supported by the by the Clinic of Fixed and Removable Prosthodontics and Dental Material Science and the Clinic of Preventive Dentistry, Periodontology and Cariology, both Center of Dental Medicine, University of Zurich, Zurich, Switzerland and by research grants of Geistlich Pharma AG, Wolhusen, Switzerland and Degradable Solutions AG, Schlieren, Switzerland. All authors do not report any conflicts of interests.

\section{Acknowledgments}

The authors would like to thank Dr. Michael Mayer, Consult AG, Bern, Switzerland for performing the statistical analysis. 


\section{References}

Amler, M. H., Johnson, P. L. \& Salman, I. (1960) Histological and histochemical investigation of human alveolar socket healing in undisturbed extraction wounds. Journal of the American Dental Association 61: 32-44.

Araujo, M., Linder, E., Wennstrom, J. \& Lindhe, J. (2008) The influence of bio-oss collagen on healing of an extraction socket: An experimental study in the dog. The International journal of periodontics \& restorative dentistry 28: 123-135.

Botticelli, D., Berglundh, T. \& Lindhe, J. (2004) Hard-tissue alterations following immediate implant placement in extraction sites. Journal of clinical periodontology 31: $820-828$.

Fickl, S., Schneider, D., Zuhr, O., Hinze, M., Ender, A., Jung, R. E. \& Hurzeler, M. B. (2009) Dimensional changes of the ridge contour after socket preservation and buccal overbuilding: An animal study. Journal of clinical periodontology 36: 442-448.

Fickl, S., Zuhr, O., Wachtel, H., Bolz, W. \& Huerzeler, M. (2008) Tissue alterations after tooth extraction with and without surgical trauma: A volumetric study in the beagle dog. Journal of clinical periodontology 35: 356-363.

Horvath, A., Mardas, N., Mezzomo, L. A., Needleman, I. G. \& Donos, N. (2013) Alveolar ridge preservation. A systematic review. Clinical oral investigations 17: 341363. 
Jung, R. E., Philipp, A., Annen, B. M., Signorelli, L., Thoma, D. S., Hammerle, C. H., Attin, T. \& Schmidlin, P. (2013) Radiographic evaluation of different techniques for ridge preservation after tooth extraction: A randomized controlled clinical trial. Journal of clinical periodontology 40: 90-98.

Pietrokovski, J. \& Massler, M. (1967) Alveolar ridge resorption following tooth extraction. The Journal of prosthetic dentistry 17: 21-27.

Schneider, D., Grunder, U., Ender, A., Hammerle, C. H. \& Jung, R. E. (2011) Volume gain and stability of peri-implant tissue following bone and soft tissue augmentation: 1-year results from a prospective cohort study. Clinical oral implants research 22: 28-37.

Tan, W. L., Wong, T. L., Wong, M. C. \& Lang, N. P. (2012) A systematic review of post-extractional alveolar hard and soft tissue dimensional changes in humans. Clinical oral implants research 23 Suppl 5: 1-21.

Thalmair, T., Fickl, S., Schneider, D., Hinze, M. \& Wachtel, H. (2013) Dimensional alterations of extraction sites after different alveolar ridge preservation techniques - a volumetric study. Journal of clinical periodontology 40: 721-727.

Thoma, D. S., Jung, R. E., Schneider, D., Cochran, D. L., Ender, A., Jones, A. A., Gorlach, C., Uebersax, L., Graf-Hausner, U. \& Hammerle, C. H. (2010) Soft tissue volume augmentation by the use of collagen-based matrices: A volumetric analysis. Journal of clinical periodontology 37: 659-666. 
Vignoletti, F., Matesanz, P., Rodrigo, D., Figuero, E., Martin, C. \& Sanz, M. (2012) Surgical protocols for ridge preservation after tooth extraction. A systematic review. Clinical oral implants research 23 Suppl 5: 22-38. 


\section{Figure legends}

Figure 1. Labial (left) and lateral view (right, from distal) of a digitized model before extraction of tooth 11 (yellow) and 6 months after treatment and healing (green). The orange and yellow lines indicate the tooth axis and the mucogingival contour line, respectively. The dotted yellow line encompasses the area of interest. The lowest images show the areas of interest used for volumetric analysis after superimposition.

Figure 2. Boxplots showing mean horizontal distances of alveolar ridge contour changes between time points. A) ß-tricalcium-phosphate-particles with a polylactid coating (ß-TCP), B) demineralized bovine bone mineral with $10 \%$ collagen covered with a collagen matrix (DBBM-C/CM), C) DBBM with $10 \%$ collagen covered with an autogenous soft tissue punch graft (DBBM-C/PG), D) spontaneous healing (control).

Figure 3. Boxplots showing horizontal bone alterations from initial alveolar crest as measured by CBCT (Jung et al. 2013). A) ß-tricalcium-phosphate-particles with a polylactid coating ( $ß-\mathrm{TCP}), \mathrm{B})$ demineralized bovine bone mineral with $10 \%$ collagen covered with a collagen matrix (DBBM-C/CM), C) DBBM with $10 \%$ collagen covered with an autogenous soft tissue punch graft (DBBM-C/PG), D) spontaneous healing (control). 